(C) 2012 IEEE. Personal use of this material is permitted. Permission from IEEE must be obtained for all other uses, in any current or future media, including reprinting/republishing this material for advertising or promotional purposes, creating new collective works, for resale or redistribution to servers or lists, or reused of any copyrighted component of this work in other works. Published in the Proceedings of the IEEE International Geoscience and Remote Sensing Symposium, Munich,

Germany, July 22-27, 2012, pp. 5993-5996.

\title{
GENERATING GROUND REFERENCE DATA FOR A GLOBAL IMPERVIOUS SURFACE SURVEY
}

\author{
James C. Tilton ${ }^{1}$, Eric Brown de Colstoun ${ }^{1}$, Robert E. Wolfe ${ }^{1}$ Bin Tan $^{2}$ and Chengquan Huang ${ }^{3}$ \\ ${ }^{1}$ NASA GSFC, ${ }^{2}$ Earth Resources Technology, Inc., and ${ }^{3}$ University of Maryland
}

\begin{abstract}
We are developing an approach for generating ground reference data in support of a project to produce a $30 \mathrm{~m}$ impervious cover data set of the entire Earth for the years 2000 and 2010 based on the Landsat Global Land Survey (GLS) data set. Since sufficient ground reference data for training and validation is not available from ground surveys, we are developing an interactive tool, called HSegLearn, to facilitate the photo-interpretation of 1 to $2 \mathrm{~m}$ spatial resolution imagery data, which we will use to generate the needed ground reference data at $30 \mathrm{~m}$. Through the submission of selected region objects and positive or negative examples of impervious surfaces, HSegLearn enables an analyst to automatically select groups of spectrally similar objects from a hierarchical set of image segmentations produced by the HSeg image segmentation program at an appropriate level of segmentation detail, and label these region objects as either impervious or nonimpervious.
\end{abstract}

Index Terms - Image Processing, Image Segmentation, Geography, Urban Areas.

\section{INTRODUCTION}

We are engaged in a project to produce a $30 \mathrm{~m}$ impervious cover data set of the entire Earth for the years 2000 and 2010 based on the Landsat Global Land Survey (GLS) data set. The GLS data from Landsat provide an unprecedented opportunity to map global urbanization at this resolution for the first time, with unprecedented detail and accuracy. Moreover, the spatial resolution of Landsat is absolutely essential to accurately resolve urban targets such as buildings, roads and parking lots. Finally, with GLS data available for the $1975,1990,2000$, and 2005 time periods, and soon for the 2010 period, the land cover/use changes due to urbanization can now be quantified at this spatial scale as well.

Our approach works across spatial scales using very high spatial resolution commercial satellite data to both produce and evaluate continental scale products at the $30 \mathrm{~m}$ spatial resolution of Landsat data. We are developing continental scale training data at $1 \mathrm{~m}$ or so resolution and aggregating these to $30 \mathrm{~m}$ for training a regression tree algorithm. Because the quality of the input training data are critical, we have developed an interactive software tool, called HSegLearn, to facilitate the photo-interpretation of high resolution imagery data, such as Quickbird or Ikonos data, into an impervious versus non-impervious map. Previous work has shown that photo-interpretation of high resolution data at 1 to $2 \mathrm{~m}$ spatial resolution will generate an accurate $30 \mathrm{~m}$ resolution ground reference when coarsened to that resolution. Since this process can be very time consuming when using standard clustering classification algorithms, we are looking at image segmentation as a potential avenue to not only improve the training process but also provide a semi-automated approach for generating the ground reference data.

We are developing a photo-interpretive tool, HSegLearn, which takes as its input a hierarchical set of image segmentations produced by the HSeg image segmentation program $[1,2]$. HSegLearn lets an analyst specify pixel locations as being either positive or negative examples, and displays a classification of the study area based on these examples. For our study, the positive examples are examples of impervious surfaces and negative examples are examples of non-impervious surfaces. HSegLearn searches the hierarchical segmentation from HSeg for the coarsest level of segmentation at which selected positive example locations do not conflict with negative example locations and labels the image accordingly. The negative example regions are always defined at the finest level of segmentation detail. The resulting classification map can be then further edited at a region object level using the previously developed HSegViewer tool [3].

After providing an overview of the HSeg image segmentation program, we provide a detailed description of the HSegLearn software tool. We then give examples of using HSegLearn to generate ground reference data.

\section{THE HSEG IMAGE SEGMENTATION PROGRAM}

A popular approach for performing image segmentation is best merge region growing. An early example is the Hierarchical StepWise Optimization (HSWO) approach of Beaulieu and Goldberg [4]. HSWO is an iterative form of region growing, in which the iterations consist of finding the 
most optimal or best segmentation with one region less than the current segmentation.

In complex scenes, such as remotely sensed images of the Earth, objects with similar spectral signatures (e.g., lakes, agricultural fields, buildings, etc.) appear in spatially separated locations. In such cases, it is useful to aggregate these spectrally similar but spatially disjoint region objects together into groups of regions objects that we call region classes. This aggregation may be performed as a postprocessing step. However, best merge region growing, as exemplified by HSWO, may be modified to integrate this aggregation directly into the region growing process. This is the basis of the Hierarchical Segmentation (HSeg) algorithm.

HSeg produces a segmentation hierarchy a set of several image segmentations at different levels of detail in which the segmentations at coarser levels of detail can be produced from simple merges of regions at finer levels of detail. This hierarchy may be useful for applications that require different levels of image segmentation details depending on the characteristics of the particular image objects segmented. An important feature of a segmentation hierarchy that distinguishes it from most other multilevel representations is that the segment or region boundaries are maintained at the full image spatial resolution for all levels of the segmentation hierarchy.

The key unique aspect of HSeg is its tight intertwining of region growing segmentation, which produces spatially connected region objects, with non-adjacent region object aggregation, which groups sets of region objects together into region classes. HSegLearn takes advantage of this region object classification to extend its positive and negative example labeling throughout the entire image based on just a few examples.

\section{THE HSEGLEARN PHOTO- INTERPRETATION TOOL}

HSegLearn provides a convenient graphical user interface for designating region classes from the HSeg segmentation hierarchy as positive and negative example region classes. One way an analyst can do this is by selecting a pixel location in a display panel showing an RGB rendition of the image data or in a display panel of the current image labeling, and having HSegLearn highlight (in yellow) the region class containing the selected pixel. The analyst can either then select another region class for highlighting, or submit the currently highlighted region class as a positive or negative example class. Another way an analyst can highlight region classes is by selecting the "Circle Region of Interest" option on one of the display panels and circling an area in the display panel. HSegLearn will then highlight every region class contained within the circled area. The analyst can either then select another region class or group of region classes for highlighting, or submit the currently highlighted region classes as positive or negative example classes.

Region classes submitted as negative examples are displayed in red at the finest resolution of the segmentation hierarchy. However, region classes submitted as positive examples are displayed in green at the coarsest resolution of the segmentation hierarchy at which the region class spatial extent does not overlap any submitted negative example region class at the finest resolution of the segmentation hierarchy. For the case for which a region class is submitted as both a positive and negative example, this region class is designated as ambiguous and colored purple in the display. An ambiguous class designation can be overridden by a subsequent resubmission as either a positive or negative example class.

\section{GENERATING GROUND REFERENCE DATA WITH HSEGLEARN}

In this section we provide an example of the generation of ground reference data with the HSegLearn photointerpretation tool. HSegLearn provides a graphical user interface (GUI) (Fig. 1) through which an analyst can bring up a display of an $\mathrm{RBG}$ rendition of the image data and a display of the current labeling of the scene (initially blank).

Our test scene is a $5512 \times 4648$ 4-band pan-sharpened Ikonos image from the Centralia, MO area at $1 \mathrm{~m}$ spatial resolution. A portion of that scene is displayed in Fig. 2(a). In our example, we first click on a pixel in the middle of a dark roof and the bottom of the image and highlight it by clicking on the button "Click Here to Highlight the Region Class Located at the Last Left Mouse Button Click." The result after "selecting" this region class as a "positive example" is shown in Fig. 2(b).

After we "submit" the selected region class as a "positive example," HSegLearn searches the HSeg segmentation hierarchy for the coarsest segmentation that contains this dark roof that also does not conflict with any region classes submitted as negative examples. Since we haven't submitted any negative examples yet, HSegLearn returns the coarsest possible segmentation from the segmentation hierarchy, as displayed in Fig. 3(a).

We now need to provide HSegLearn with some negative examples. We can do this by circling a region of interest in, for example, an agricultural field, as shown in Fig. 3(b), and the highlighting and selecting the HSeg region classes (at the most detailed segmentation level in the segmentation hierarch) contained in the region of interest as negative examples. We can also highlight and select individual region classes as negative examples in the same way we highlighted and selected the dark roof positive example. The result after highlighting and selecting several HSeg region classes as negative examples is shown in Fig. 4(a). 


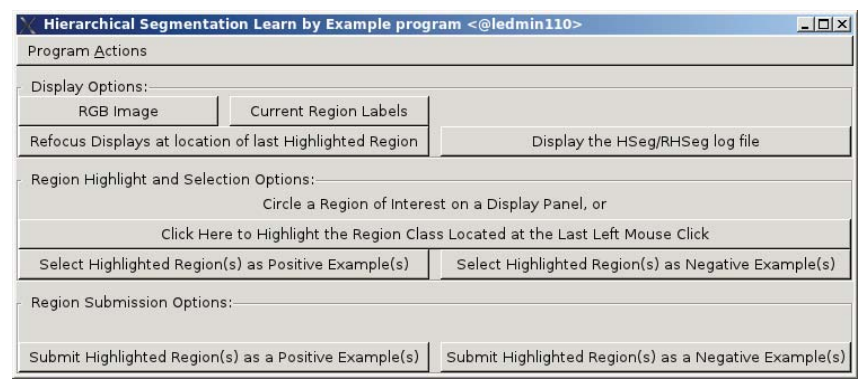

Figure 1. An example of the HSegLearn graphical user interface.

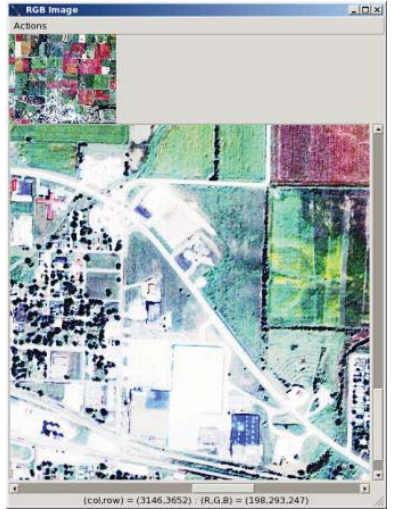

(a)

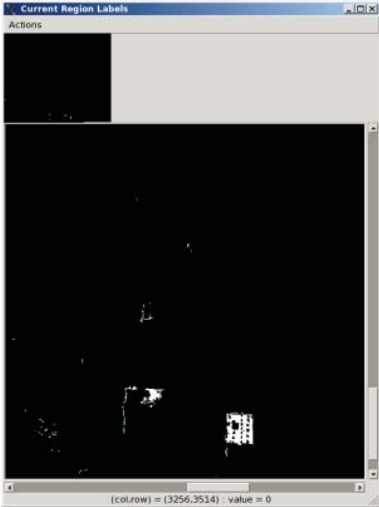

(b)
Figure 2. (a) RGB rendition of a portion of an Ikonos image. (b) The current region labeling after selecting the HSeg region class associate with a dark roof.

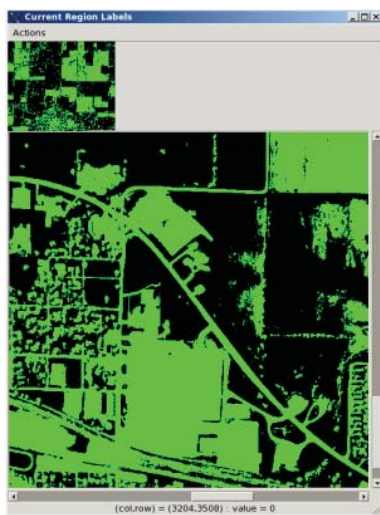

(a)

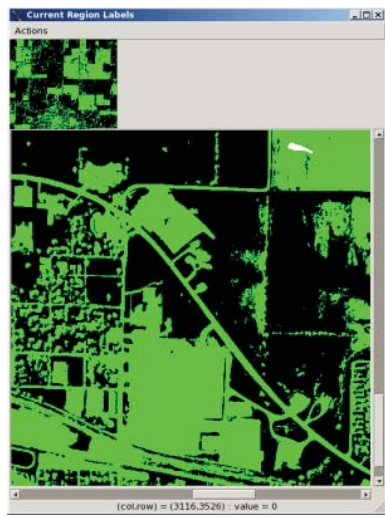

(b)
Figure 3. (a) The current region labeling after submitting the HSeg region class associate with a brown roof as a positive example (with no negative examples yet provided). (b) The current region labeling after circling a region of interest in an agricultural field.

We now submit our negative examples to HSegLearn. When we do this, HSegLearn adjusts the selection of the hierarchical segmentation level of the previously submitted positive example(s) so that the positive example labeling (a)

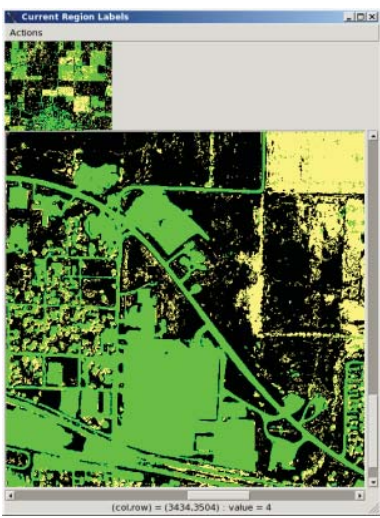

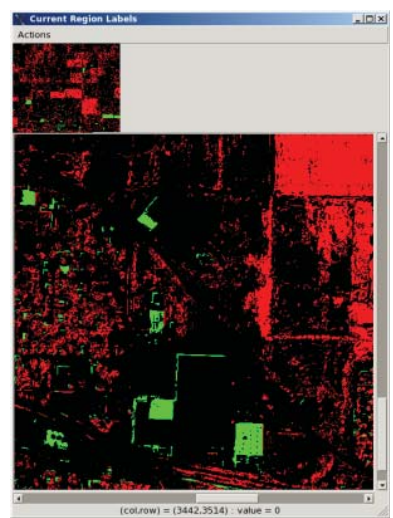

(b)
Figure 4. (a) The current region labeling after selecting (and not yet submitting) several negative examples. (b). The current region labeling after submitting several negative examples.

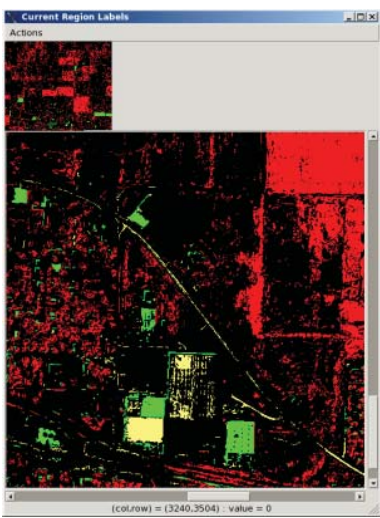

(a)

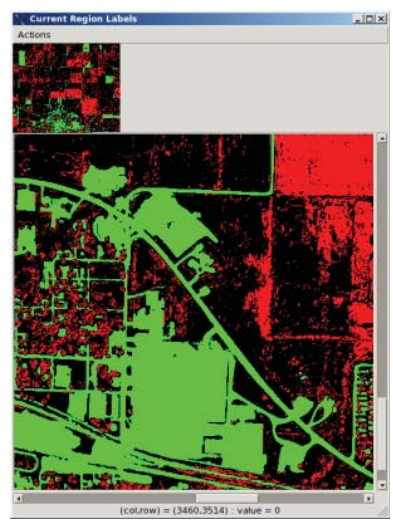

(b)
Figure 5. (a) The current region labeling after several positive examples of impervious surfaces are selected (roofs and parking lots). (b). The current region labeling after the additional positive examples are submitted to HSegLearn.

does not conflict with the submitted negative examples. The result is shown in Fig. 4(b). Note that most of the area previously highlighted as impervious (green) is now left unlabeled.

To increase the labeling of positive examples of impervious surfaces we must provide HSegLearn with more positive examples. The result of selecting another roof and a parking lot as positive examples is displayed in Fig. 5(a). Note that the selected region classes are highlighted per the finest segmentation level in the HSeg segmentation hierarchy. After we submit these positive examples, HSegLearn, searches the HSeg segmentation hierarchy for the coarsest segmentation levels in the HSeg segmentation hierarchy for labeling the image, as shown in Fig. 5(b). The result is a very complete and accurate labeling of this portion of the image as impervious (green) versus nonimpervious (red or black). 


\section{DISCUSSION}

The HSegLearn tool solves a key problem we have encountered with other approaches we have attempted to use for generating ground reference data from high spatial resolution imagery. This key problem was how to define the most effective level of segmentation or clustering detail. When too few clusters or region segments were selected, the classes of interest could not be separated, but when too many clusters of region segments were selected, the labeling of the classes of interest became very inefficient. HSegLearn automatically selects the most effective level of segmentation detail for labeling the classes of interest (for a binary positive class/negative class labeling problem) based on a simple selection of positive and negative examples by an analyst.

The effectiveness HSegLearn's automatic selection of level of segmentation detail is demonstrated in the example discussed in the previous section. With the selection of just a few positive example region classes, along with a judicious selection of a few negative example region classes, a very accurately labeling of the image into impervious versus non-impervious surfaces is quickly obtained.

\section{ACKNOWLEGMENT}

We would like to acknowledge the helpful feedback we received from other participants in our project, which resulted to several improvements to the HSegLearn tool: Sarah E. Smith, Universities Space Research Association, and Md Aminul Islam, University of Maryland.

\section{REFERENCES}

[1] J. C. Tilton, "Image segmentation by region growing and spectral clustering with a natural convergence criterion," Proc. of IGARSS'98, Seattle, WA, pp. 1766-1768, 1998.

[2] J. C. Tilton, Y. Tarabalka, P. M. Montesano, and E. Gofman, "Best merge region growing with integrated non-adjacent region object aggregation," IEEE Trans. Geosci. Remote Sens., vol. 50, no. 10, Oct. 2012.

[3] J. C. Tilton, RHSeg User's Manual: Including HSWO, HSeg, HSegExtract, HSegReader and HSegViewer, version 1.55, available via email request to James.C.Tilton@nasa.gov, January 4, 2012.

[4] J.-M. Beaulieu and M. Goldberg, "Hierarchy in picture segmentation: A stepwise optimal approach," IEEE Trans. Pat. Anal. Machine Intell., vol. 11, no. 2, pp. 150-163, Feb. 1989. 